\title{
How Jordanian and Syrian Youth Conceptualise Their Sexual and Reproductive Health Needs: A Visual Exploration Using Concept Mapping
}

\section{Citation}

Gausman, Jewel, Areej Othman, Insaf Daas, Iqbal Hamad, Maysoon Dabobe, and Ana Langer. How Jordanian and Syrian youth conceptualise their sexual and reproductive health needs: a visual exploration using concept mapping. Culture, Health \& Sexuality. February 27, 2020. DOI: 10.1080/13691058.2019.1698769

\section{Permanent link}

http://nrs.harvard.edu/urn-3:HUL.InstRepos:42556230

\section{Terms of Use}

This article was downloaded from Harvard University's DASH repository, and is made available under the terms and conditions applicable to Open Access Policy Articles, as set forth at http:// nrs.harvard.edu/urn-3:HUL.InstRepos:dash.current.terms-of-use\#OAP

\section{Share Your Story}

The Harvard community has made this article openly available.

Please share how this access benefits you. Submit a story.

\section{Accessibility}


This is a post-peer-review, pre-copyedit version of an article published in Culture, Health And Sexuality. The final authenticated version is available online at:

https://doi.org/10.1080/13691058.2019.1698769

How Jordanian and Syrian Youth Conceptualize their Sexual and Reproductive Health Needs: A Visual Exploration using Concept Mapping

Jewel Gausman*a, Areej Othman ${ }^{b}$, Insaf Daas ${ }^{c}$, Iqbal Hamad $^{d}$, Maysoon Dabobe ${ }^{d}$, Ana Langer ${ }^{a}$

Women \& Health Initiative, Department of Global Health and Population, Harvard TH Chan School of Public Health, Boston, MA, USA ; Maternal Child Health Nursing Department, School of Nursing, University of Jordan, Amman, Jordan ; Center for Women's Studies, University of Jordan, Amman, Jordanc; Jordanian Hashemite Fund for Human Development (JOHUD), Amman, Jordan ${ }^{d}$

*Corresponding Author: Jewel Gausman Email: jgausman@mail.harvard.edu

\begin{abstract}
Young people in Jordan are caught between the rapid social change that has encompassed the Middle East and the pressure to adhere to the strict norms and values that have defined previous generations, especially with regard to sexual and reproductive health. This study seeks to understand how Jordanian and Syrian adolescents conceptualise their concerns, needs and challenges with respect to their developing sexuality and reproductive health, while offering a comparative perspective by nationality and gender. 271 young people between the ages of 15 and 19 participated in the study. Data were generated using an interactive concept mapping approach. Data collection included brainstorming, pile sorting and rating to create a visual map that was interpreted by the participants. The results of this study show that both Jordanian and Syrian adolescents have a complex understanding of sex, sexuality and reproductive health that they define through broad conceptual spheres which include health, economic and social issues. Differences by gender highlight the conflict between traditional norms and changing social expectations. Both Jordanian and Syrian participants identified the need for diverse informational resources. For Syrians, the results emphasise how the hardship has influenced sexual and reproductive through a focus on early marriage and need for economic stability.
\end{abstract}

Keywords: Sexual and reproductive health; adolescents; refugees, Syria; Jordan 


\section{Introduction}

Young people in Jordan are caught at a crossroads between the rapid social change brought about by the economic turmoil, political instability and globalisation that has characterised much of the Middle East over the last decade, combined with the profound pressure they face to conform to the often strict social and cultural norms that defined previous generations. Traditional and religious norms value the preservation of honour (Uhlmann 2005) and prohibit sexual relationships outside of marriage (Mrayan and Cornish 2015). Both men's and women's behaviour is often closely monitored, and transgressions from acceptable sexual behaviour may be punished with violence or death (Al Gharaibeh 2016). Discussing sex-related issues is generally considered to be socially unacceptable. Unsanctioned sexual activity among young people often occurs in the context of extreme secrecy and with limited information on how to protect oneself from risk (DeJong et al. 2005; Obermeyer, Bott, and Sassine 2015).

While the average age of marriage overall is increasing in Jordan, rates of child marriage have increased among some population sub-groups (Hikmat 2017). Data indicate that between 2011 and 2015 the percentage of marriages involving a woman under the age of 18 years rose from $12 \%$ to $34.6 \%$ among Syrian refugees (Higher Population Council 2017). At the same time, more young people are pursuing educational and economic opportunities before marriage, which may increase the need for sexual and reproductive health (SRH) services as the gap between sexual maturation and marriage grows (DeJong et al. 2007; DeJong et al. 2005). In Jordan, data on premarital sexual activity is extremely limited and only one study conducted in 2001 could be found in the literature estimating that $7 \%$ of university students and $4 \%$ of the general population between the ages of 15 and 30 years engage in premarital sexual activity (Schoemaker et al. 2001).

The Syrian conflict has had a profound impact on how youth in Jordan transition to adulthood. Jordan is currently home to more than 1.2 million Syrian refugees (Department of Statistics [Jordan] 2016). The crisis has exacerbated ongoing economic hardships as a result of increased competition for limited employment opportunities (UNICEF 2015) and many young people are forced to drop out of school. Some refugee families believe that early marriage protects their daughters in what is perceived as an unsafe environment where sexual harassment and assault, rape and prostitution are thought to be relatively common, while also providing families with possible economic benefits (Sexual and Gender-Based Violence Sub-Working Group 2015).

The sensitive cultural context surrounding young people's SRH in Jordan renders its study challenging (Okour and Badarneh 2011). Across the Middle East, the limited SRH data available on youth is often interpreted as a lack of need (Ercevik Amado 2009). To address this important research gap, this study seeks to understand how Jordanian and Syrian adolescents understand their concerns, needs, and challenges with respect to their developing sexuality and reproductive health, while offering a comparative perspective by both nationality and gender.

\section{Methods}

This study uses a qualitative research methodology called concept mapping (CM) (Burke et al. 2005). CM engages participants in an iterative, participatory data collection process that occurs 
during several sequential group sessions (described below). Data analysis is interspersed between data collection sessions and encourages active involvement by participants. The process ultimately results in the development of a visual map depicting how a group views a particular topic. A detailed study protocol outlining all study procedures and analysis has been published elsewhere (Gausman, Othman, Hamad, et al. 2019). This study received ethical approval from the institutional review boards at the Harvard T.H. Chan School of Public Health's Office of Human Research Administration and the University of Jordan School of Nursing Institutional Review Board.

Participants were recruited by convenience at community centres in four cities across Jordan: Amman, Irbid, Mafraq, and Zarqa. To be eligible, participants had to be Jordanian or Syrian and aged between 15-19 years. Informed consent was obtained for individuals 18 years and above. Informed assent and written parental permission were obtained for participants under the age of 18 .

In each community, participants were assigned to one of four group sessions corresponding to sex and national origin. Each group met once weekly for three weeks with the same participants. Session facilitators were community outreach workers in the same communities as study participants. They were between 20 and 35 years of age and were the same sex as the participants in the groups to which they were assigned. In the first group session, participants first worked in pairs and then as a large group to brainstorm items related to their "sexual and reproductive health concerns, challenges, needs, or fears." After the first session, the study team consolidated the items from all groups into two master lists, separated by gender. During the second session, participants were given notecards containing all items from the master list. They worked in pairs to organise the items into meaningful piles and to generate a name for each pile that captured the relationship between the items. Participants then individually rated each item in terms of its importance to their SRH on a scale of 1 (not important) to 5 (extremely important).

Data from the second session were analysed using hierarchical cluster analysis (Kane and Trochim 2007). The analysis was performed using the R package Vegan (Oksanen et al. 2016 ). The resulting visual maps represent the preferred spatial configuration of the aggregated groupings of items that represent higher-level concepts. Separate maps were generated for young women and young men. The physical distance between any two points or clusters on the map represents the degree of similarity between the items so that items/clusters located near to each other are more similar than items that appear further apart (Campbell and Salem 1999). Cluster names were selected based on themes identified in participant data.

Basic statistics were calculated for each item, including the item's bridging value. The bridging value indicates how similar an item is to the other items within a cluster (Jackson and Trochim 2002), with values ranging from 0 to 1 . A low bridging value $(<0.25)$ indicates that an item is conceptually similar to the other items in its cluster, while a high bridging value $(>0.75)$ indicates that an item was often sorted with items located in other clusters (Brown et al. 2008). A lower mean bridging value for a cluster suggests greater homogeneity between items while a higher bridging value is indicative of a more heterogeneous concept that is less well-defined (Iris, Ridings, and Conrad 2009). Bridging values are useful in determining the number of clusters to 
include in the final map. In a well-developed map, few clusters will have high bridging values thereby indicating that the concepts identified are relatively distinct from one another.

In the final session, participants were asked to interpret the maps and discuss the importance of rankings, first in small groups and then together as a large group. Discussions were recorded, transcribed and analysed thematically.

\section{Results}

In total, 271 young people aged 15-19 years participated in the study across the four study sites, with an average of 30 men (ranging from 22-39 participants) and 37.5 women (ranging from 3441 participants) per group. Table 1 describes the background characteristics of the participants. The majority were aged 15-16 years and were unmarried. Approximately half of the young women (49\%) were currently enrolled in school while most young men (60\%) reported not attending school.

[Insert Table 1 near here]

Overall, attrition during the study was low. Among young women, attendance across all sites was $91 \%$ and $82 \%$ in the second and third sessions respectively, while for young men, attendance was $98 \%$ and $95 \%$ in the second and third sessions respectively.

Two-dimensional cluster maps are presented in Figures 1 (young women) and 2 (young men). The young women's map contains 101 items in nine clusters. The young men's map contains 76 items in seven clusters.

\section{Young women's cluster map}

On the young women's map, the clusters located on the left-hand side tend to relate to SRH in a positive way. Cluster 1 , "Wishes," includes changes young women wished they could see in their communities, such as young men treating girls and young women with more respect, improved safety, and increased openness and acceptance. As explained by one Jordanian girl in Irbid, an "open-minded society" is important to SRH because it improves awareness and respect for women's rights. She explained that currently, "if something happens to a girl, society does not blame the man, they always blame the girl... whether she was forced or by choice, they always blame the girl." Similarly, another Jordanian girl in Irbid explained, "they kill the girl for honour and nothing happens to the guy." A Syrian girl in Mafraq explained that, "we have a wish that society would be a little bit more civilised and more understanding... that they wouldn't keep saying: 'No, unacceptable, shameful, wrong, forbidden'... this creates psychological stress for the girl." Cluster 1 also includes items related to young women wanting a range of reliable and convenient sources of SRH information and services in their communities, including social and psychological services.

[Insert Figure 1 near here] 
Cluster 5, "Obtaining information from parents and other credible sources," includes items that relate to formal and informal educational resources. The items in this cluster emphasise that young women not only want improved SRH education for themselves, but they want education for others in their community as well, such as peers, teachers, school leader, and parents, so that they have trusted resources on which they can rely. The importance of strong parent-child relationships emerges from within the cluster, especially between mothers and daughters. As one Syrian girl stated, "[with regard to] educating parents..., the mother might be too shy to tell her daughter 'one, two and three.' Some mothers do not have the confidence to discuss these matters with their daughter, fearing that it might open up further issues."

Clusters 8 and 9 are located near to each other on the young women's cluster map. "Cluster 8, "Healthy and positive marital life," focuses on developing positive marital relationships with a suitable person at a suitable age and fulfilling the perceived responsibilities of marriage, such as raising children and engaging in sexual activity. Items in the cluster also emphasise the importance of gender equity in healthy marriage. A Syrian girl in Mafraq explained that the items in Cluster 8 highlight the interconnection between how SRH, mental health, and living conditions influence the health of a marriage by focusing on the item "stability," in that "Stability is that a girl and a guy are financially, emotionally, mentally stable." Cluster 9: "family planning," is conceptually similar to Cluster 8 given its location, and includes items related to fertility and the ability to take care of children.

On the right-hand side of the map, clusters become less positive with regard to how they relate to SRH. In Cluster 4, young women identify how the conservative society in which they live negatively influences their SRH by limiting their ability to report harassment while restricting clothing choices and access to SRH services. In relation to the items in this cluster, a Syrian girl in Irbid contrasted her experience in Jordan with her understanding of what life is like in "more open societies" by saying, "sometimes a girl might be dressed in an unacceptable way...and a young man will...involuntarily comment. If you look at a foreign country, a girl might be wearing shorts and its ok, no one looks at her."

Clusters 3 and 6 both relate to marriage, although in different ways. Cluster 3, "issues related to marriage and pregnancy," includes a range of items that relate to problems in childbearing, such as a lack of SRH information, marriage to relatives, genetic diseases and family disintegration. While many of the items in this cluster are negative in meaning, a few items appear to be neutral (i.e. pre-conception and bodily changes during pregnancy). Cluster 6, "Violence and unhealthy marriage," highlights a relationship between forced and early marriage, intimate partner violence, poor SRH and mental health outcomes.

Clusters 2 and 7 contain the largest number of items. Cluster 2, "Culture of shame," encompasses items relating to normal biological and behavioural changes during puberty (i.e. acne, menstrual irregularity, shyness, and awkwardness), health-related items (i.e. psychological stressors and STIS), and culturally prohibited behaviours (i.e. same-sex sexual relationships, marital infidelity, and losing virginity before marriage). In response to the breadth of items contained in the Cluster 2, a Syrian girl in Zarqa commented that, "everything in society is considered shameful, especially for a girl." With regard to shame associated with biological factors, a Jordanian girl in Irbid explained, "for girls, when her period is late, [her] parents' get suspicious. Why is your period late? They wouldn't think she might have a problem... they go further with their thinking." The young women also included being a victim of sexual as part of 
the concept of "shame." As explained by a Syrian girl in Mafraq "With harassment...if you want to talk about the incident, they will say: shameful!" In Cluster 7, "Fears," young women identify a variety of items that relate to fears over biological processes (i.e. puberty and menstruation), social expectations (i.e. early or late pregnancy and marriage, not having children, relationships with in-laws, motherhood, giving birth to a female child), attributes of marriage (i.e. sexual relationships, having a life partner, marriage before finishing school, and economic concerns), sexual assault and rape, and social relationships with adults and other youth. A Syrian girl in Irbid reflected on how Clusters 7 and 1 are located on opposite sides of the map by saying, "when there is awareness within society, fears will decrease..."

\section{Young men's cluster map}

As with the young women's map, the young men's map is organised so that concepts related to $\mathrm{SRH}$ in a positive or negative way are located on the left- and right-hand sides, respectively. Items in Cluster 1, "Educational and informational sources," relate to the need for adequate and diverse informational resources, such as cultural centres, schools, and the internet. Cluster 7, "Needs for a safe and healthy marital life," focuses on a variety of aspects relating to satisfying a range of basic needs for a healthy family and raising children, such as the availability of housing, work, and health services. Young men also emphasised the need for information about marriage and relationships from both formal and informal sources. Cluster 6, "Puberty and adulthood," includes a variety of items that young men associate with transitioning to adulthood, including medical tests, sexual experimentation, no longer living with parents, pregnancy, and childbirth. In Cluster 4, "SRH information, needs and concerns," young men focused on a variety of items that primarily relate to sexual activity, including biological aspects, (i.e. concerns about male and female genitalia), emotional aspects (i.e. self-confidence and lust), behavioural aspects (i.e. condom use, sexual exercise, self-confidence), attributes of male-female relationships, and the need for sexuality education. As emphasised by one Jordanian boy from Irbid, "we should have cultural centres to educate the public."

[Insert Figure 2 about here]

Clusters 2, 3, and 5 focus on concepts related to SRH that young men believe to be negative or harmful. Cluster 5, "Factors influencing $\mathrm{SRH}$," includes items that relate primarily to social and behavioural issues (i.e. the use of drugs, steroids and Viagra, engaging in prohibited behaviours, early pregnancy and social pressure), but also concerns about relationship incompatibility and shyness. One Syrian boy in Irbid indicated that "shyness or embarrassment prohibits the initiation of relationships," while a young Jordanian man in Irbid explained that "drug [use] leads to impotence."

In Cluster 3, "Fears," young men identified fears that pertain to biological issues (i.e. erectile dysfunction, disease, and consequences of sexual relationships), but also fears pertaining to cultural and social aspects of sexual behaviour (i.e. adultery, parents, and quarrels). Several items relate to fears of being the victim of sexual assault, such as "fear of the night," "fear of older boys," and "fear of suspicious places." Such fears were echoed among both Jordanians and Syrians. A Syrian young man in Irbid commented that some older boys are believed to sexually 
assault and rape younger boys in the community, while another indicated that "fear of the night means fear of harassment, rape and older youth."

Cluster 2, "Socially unacceptable behaviours and shame," includes items related to pubertal development (i.e. masturbation, sperm, etc.) and sexual images (i.e. pornography and movies). Items that relate to sexually transmitted infections, same-sex sexual relationships, sexual assault and rape are also associated with shame.

Table 2 presents the mean cluster importance and bridging value for young men and women, stratified by the respondent's nationality. Importance and bridging values for each individual item can be found in online supplemental Table 1.

Jordanian young women ranked "good parenting" (3.64), "marriage at an appropriate age" (3.54), and "awareness and counselling by mother" (3.53) as the most important individual items, while Syrian young women ranked "marriage at an appropriate age" (3.39), "psychological stressors" (3.34), and "understand the responsibilities of marriage" (3.28) as the top three items. In terms of the overall cluster score, the most important cluster among Jordanian young women was "Cluster 9: Family planning," while for Syrian young women, it was "Cluster 8: Healthy and positive marital life." The mean bridging values calculated for each of the nine clusters indicate fairly strong homogeneity between items within each cluster. Mean bridging values range from 0.24 in "Cluster 7: Fears" to 0.60 in "Cluster 4: Conservative Society."

[Insert Table 2 Near Here]

Jordanian young men ranked "fear of parents' anger" (3.29), "fear of adultery" (3.23), "availability of work" (3.22) and "healthy food" (3.12), while Syrian young men ranked "fear of infectious disease" (3.12), "availability of work" (3.12) and "fear of parents' anger" (3.06) as the most important items. Of note, $37 \%$ of young men ranked "homosexuality," $32 \%$ ranked "having sex with someone of the same sex," 44\% ranked "sexual assault" and $25 \%$ ranked "rape" as either somewhat important or very important to their SRH. According to mean item scores, Jordanian young men ranked "Cluster 7: Needs for a safe and healthy marital life" as the most important cluster overall, while Syrian young men ranked "Cluster 3: Fears" as the most important cluster.

Cluster 1: "Educational and informational sources" exhibits a high bridging value which suggests that the items contained in this cluster may also relate to those in other clusters. Conversely, Cluster 9: "Socially unacceptable behaviours and shame" (0.09), Cluster 3: "Fears" (0.24), and Cluster 4: "SRH information, needs, and concerns" (0.24) exhibit low bridging values meaning that they are relatively homogenous in meaning.

\section{Discussion}

The results of this study show that Jordanian and Syrian adolescent men and women have a complex understanding of sex, sexuality and reproductive health that they define through broad conceptual spheres that encompass a wide range of health, economic and social issues. The concept maps provide a visual illustration of the strong influence that gender norms have on how young people in Jordan experience sexual maturation, while highlighting the conflict between traditional social expectations and the increasingly powerful global influence that has emerged 
across the Middle East. Finally, for Syrian young women, the results emphasise how SRH overlaps with mental health, especially in relation to issues such as child marriage, war and displacement.

Broadly speaking, the young men and women in this study identified similar concepts with regard to how they understand and define issues related to their $\mathrm{SRH}$. Participants emphasised the need for more robust and trustworthy educational resources outside of the clinical setting. Previous research in Jordan indicates that existing health services do not adequately address the SRH needs of youth (DeJong et al. 2007) The maps suggest that such resources should address a wide range of topics, including puberty, marriage, male-female interaction, spousal and family relationships, mental health and supporting a family. Expanding the availability of educational programmes for youth in Jordan could help reduce the shame and fear associated with such topics. Nascent attempts are underway to introduce comprehensive sexual education into Jordanian schools and universities (Ali et al. 2018); however, sexual education remains a highly contentious matter. Considerable effort is still required to sensitise policy makers, community and religious leaders and other key stakeholders of the value of providing SRH information and services to youth.

The concepts of "shame" and "fear" feature prominently in both the young men's and women's maps as large and diverse constructs that are deeply embedded in the way Jordanian and Syrian young people understand sex and sexuality. Despite the diversity of items included within these clusters, young men and women view the clusters as relatively homogenous concepts, given their low bridging values. In both maps, the concepts of "shame" and "fear" include items that relate to normal biological and behavioural changes associated with puberty, but also reflect the influence of social norms that dictate acceptable sexual behaviours and condemn those thought to be socially unacceptable. Deviation from normative sexual behaviour can carry harsh consequences, including social ostracisation and honour killing (Dialmy 2005). Interventions to destigmatise normal sexual development and address the harmful norms surrounding sexual assault (World Health Organization 2012) may encourage improved careseeking among young people.

Despite high-level conceptual similarities between the young men's and women's maps, individual items that comprise the clusters diverge according to gender. For example, young men associated parents with fear, while young women identify parents as being a source of SRH information. Throughout much of the Middle East, cultural tradition emphasises the importance of a strong family unit (DeJong et al. 2005). A previous study in Jordan found that young people consider their parents to be a trusted source of SRH information, but youth believe their parents to be ill-equipped for such discussions (Khalaf, Moghli, and Froelicher 2010). As per the results of this study, youth want educational programmes to address their parents' limited knowledge. As young people suffer the consequences of their parents' misinformation about SRH, educating parents could be an important opportunity to reduce the shame and stigma surrounding SRH. The results of this study suggest that interventions to engage parents should consider genderrelated differences in parent-child relationships, strengthen parent-child trust and improve parental knowledge about SRH so that youth can approach their parents without fear of repercussion.

Items contained in the constructs relating to marriage also differ by gender. For example, gender-based violence (GBV) features prominently in the young women's map. GBV is widely perpetrated and accepted in Jordan (Department of Statistics [Jordan] and ICF International 
2013). Married adolescents face a higher risk of experiencing violence and other adverse health outcomes than those married later (Clark 2017), and may have little ability to identify their symptoms, seek treatment, or advocate for medical care, as the same conservative cultural norms that support child marriage also limit women's independent movement (Gausman, Othman, Amawi, et al. 2019). In the young men's map, "Needs for a safe and healthy marital life" focuses on expectations of men in Jordanian society, including finding work and housing, and providing a safe and secure life for children. Jordan's economy has struggled under the weight of both the refugee crisis and fiscal reform; currently, the official unemployment rate hovers around 18\% (Department of Statistics [Jordan] 2019) and many young men are unable to satisfy social expectations related to employment.

Finally, the young women's map highlights a tension between the desire to satisfy traditional gender roles and expectations that co-exist with a mounting desire for change. In Cluster 7: "Fears," for example, young women express fears related to conforming to social norms regarding childbearing, such as not wanting to have a child at too old of an age for fear of social repercussion, but at the same time, they also fear not being able to complete their studies before marriage. A similar tension is also illustrated amongst the items included in Cluster 4: "Conservative society" and Cluster 1: "Wishes," In Cluster 4, young women identify a relationship between conservative cultural norms and poor access to SRH services, while in Cluster 1, young women link social change with improved SRH information and service availability.

The young men's map highlights the influence of normative gender roles with items that depict traditional, hegemonic representations of masculinity. In particular, the young men's map shows a preoccupation with sexual development and sexual activity. The centrality of male sexual performance to the masculine ideal emerges in the young men's map with regard to the way that items that come into conflict with dominant masculinity are assigned. A study conducted in Lebanon found that many men view infertility as emasculating (Inhorn and Wentzell 2011)-a perception that is thought to exist across the region (Dialmy 2005). Much of the discourse on sexual roles in Arab countries focuses on equating male sexual dominance with virility with masculinity (Dialmy 2005). Many of the items in the young men's map are centred on maleinitiated sexual behaviour. The results of this study are consistent with those of another study conducted with Palestinian men in Jordan, which found that young men in the study often embodied a masculinity focused on demonstrating a strong interest in sexual behaviour and control over women's behaviour and dress (Hart 2008).

In Jordan, as in much of the Middle East, same-sex sexual relationships are deeply stigmatised and often socially and religiously prohibited (Alkaiyat et al. 2014). Items relating to same-sex sexual relationships were associated with the constructs of "shame" and "fear." While there remains a tremendous gap in the literature on same-sex sexual behaviour in the Middle East, a few small, qualitative studies conducted in Oman, Morocco and a Palestinian refugee camp in Jordan have found that some adolescent boys discuss engaging in same-sex sexual activity, especially in relation to the inaccessibility of girls during puberty (Obermeyer 2000; Hart 2008).

Information on sexual minorities in Jordan is extremely limited, but national data released in 2010 estimate that $65 \%$ of men who have sex with men also have sex with women, and that condom usage and HIV testing is very limited (Mumtaz et al. 2011). In another study, participants under the age of 25 were most likely to report shame as an obstacle to obtaining condoms 
(Alkaiyat et al. 2014), thus highlighting a need for outreach and services that target young men. Between $25 \%-44 \%$ percent of young men in this study indicated that items relevant to same-sex sexual behaviour were important to their SRH. While the results do not indicate why the participants ranked these issues as important, they demonstrate a need for research and programmes to better meet the needs of sexual minority youth in Jordan. Future research and intervention should address the social context that causes young men to either contribute to the stigmatisation of homosexuality or to feel ashamed and fearful if they are engaging in such behaviour. Challenging social stigma within communities combined with sensitising and training educational and health service providers are important next steps to ensure that young people are able to obtain information on how to protect themselves from health risks (Arreola et al. 2015), although this is very controversial in the Jordanian context.

The results of this study build upon past research that has raised concerns about the prevalence of sexual violence perpetrated against men during the Syrian war and in camps and communities that host Syrian refugees (Chynoweth 2018)(Sexual and Gender-Based Violence Sub-Working Group 2015). Some non-governmental organisations in Jordan offer basic services to the male survivors of sexual assault; however, services must be expanded and capacity must be built among providers to ensure adequacy of services (Chynoweth 2018).

The results of this study also suggest that new and alternative masculinities may be emerging among youth in Jordan. In Cluster 7, for example, the young men included items relating to marriage, relationships and family planning. Traditionally, such issues are considered to be a woman's responsibility. Literature on Arab masculinities often portrays men as the violent, hyper-dominant oppressors of women (Inhorn 2012), and often does not recognise other masculinities. This study's results suggest that youth in Jordan may not conform to these traditional stereotypes and may suggest an important opening for gender transformative interventions that encourage young men to endorse and adopt more gender-equitable ideas.

Finally, this study highlights the difficult circumstances experienced by young, female Syrian refugees in Jordan. The focus on early marriage is not surprising, given the increases in early marriage observed within the Syrian refugee population in Jordan (Higher Population Council 2017). Trauma from the war in Syria and the difficult daily living situations faced by many Syrian refugees has likely had profound psychological consequences. It is estimated that more than $50 \%$ of female Syrian refugees suffer from depression or post-traumatic stress syndrome (James et al. 2014). The Syrian women in this study emphasised the interrelationship between "psychological stressors," the war, restrictive social norms and early marriage, thus highlighting the need to address the unique concerns of this population through integrated programming approaches.

\section{Strengths and limitations}

The use of $\mathrm{CM}$ as a methodology provided young people with an opportunity to define and discuss issues related to SRH in an unstructured way. Furthermore, this research approach empowered participants to interpret their own data - thus increasing the validity of the results. Despite these strengths, there are some important limitations worth noting. Participants in this study were recruited through local community centres in highly disadvantaged areas. The majority of young men in the study had dropped out of school despite education being 
compulsory through the age of 16 years in Jordan. As such, the results may not reflect the concerns of the general population. Furthermore, some participants may not have felt comfortable discussing all the topics in an open setting or understood all of the terms used, though, participants became more comfortable with sensitive topics over the course of the successive sessions (Gausman, Lloyd, et al. 2019). Finally, the prompt used for brainstorming focused primarily on deficits rather than on how SRH connects more broadly with health and wellbeing as it was thought to be most relevant to intervention. As such, the results should not be interpreted as providing a comprehensive accounting of all domains related to young people's $\mathrm{SRH}$. Future studies using the same methodology could broaden the prompt if a wider discussion is desired.

Since the Arab Spring, young people across the Middle East have become champions of social change, and some have begun to reject some of the norms espoused by previous generations in favour of more inclusive and gender-equitable ideas. The results of this study point toward important and understudied areas to explore in future research, but also opportunities for intervention to make SRH programmes more relevant to the unique needs of this population. By exploring how young people understand, question and define the issues they find most relevant to their $\mathrm{SRH}$, the results of this study offer a robust platform that could be integrated into $\mathrm{SRH}$ education programmes and health services designed to address the needs most pertinent to youth in ways that are most salient to them.

\section{Funding Information}

This work is part of the Sexual and Reproductive Health Research Programme with project number W 08.560.012, which is financed by the WOTRO Science for Global Development of the Netherlands Organisation for Scientific Research (NWO).

\section{Disclosure statement}

No conflicts of interest to report

\section{Acknowledgements}

The authors would like to thank Dania Zabalawi and Mohannad Aldiqs for their tireless efforts in data entry, translation and transcription. They also thank the data collection team at the Jordanian Hashemite Fund for Human Development for their work in conducting the concept mapping sessions, as well participants for their participation in data collection activities.

\section{References}

Al Gharaibeh, F. M. 2016. "Debating the Role of Custom, Religion and Law in 'Honour' Crimes: Implications for Social Work." Ethics \& Social Welfare 10 (2):122-39. doi: 10.1080/17496535.2016.1155632.

Ali, Reem A., Ahlam Alnatour, Karimeh Alnuaimi, Fatmeh Alzoubi, Maysa Almomani, and Areej Othman. 2018. "Effects of Interactive Teaching on University Students' Knowledge and 
Attitude Toward Reproductive Health: a Pilot Study in Jordan." Journal of multidisciplinary healthcare 11:211-21. doi: 10.2147/JMDH.S160135.

Alkaiyat, Abdulsalam, Christian Schaetti, Mohammad Liswi, and Mitchell G. Weiss. 2014. "Condom Use and HIV Testing Among Men Who Have Sex with Men in Jordan." Journal of the International AIDS Society 17 (1):18573-. doi: 10.7448/IAS.17.1.18573.

Arreola, Sonya, Glenn-Milo Santos, Jack Beck, Mohan Sundararaj, Patrick A. Wilson, Pato Hebert, Keletso Makofane, Tri D. Do, and George Ayala. 2015. "Sexual Stigma, Criminalization, Investment, and Access to HIV Services Among Men Who Have Sex with Men Worldwide." AIDS and Behavior 19 (2):227-34. doi: 10.1007/s10461-014-0869-x.

Brown, Jason D, Susan Rodger, Natalie George, D St Arnault, and Jennifer Sintzel. 2008. "Rewards of Parenting a Child with a Fetal Alcohol Spectrum Disorder." The Open Family Studies Journal 1:23-30.

Burke, Jessica G, Patricia O'Campo, Geri L Peak, Andrea C Gielen, Karen A McDonnell, and William MK Trochim. 2005. "An Introduction to Concept Mapping as a Participatory Public Health Research Method." Qualitative Health Research 15 (10):1392-410.

Campbell, Rebecca, and Deborah A. Salem. 1999. "Concept Mapping as a Feminist Research Method: Examining the Community Response to Rape." Psychology of Women Quarterly 23 (1):65-89. doi: 10.1111/j.1471-6402.1999.tb00342.x.

Chynoweth, Sarah. 2018. "We Keep it in our Heart. Sexual Violence Against Men and Boys in the Syria Crisis." In.: UNHCR.

Clark, C. J.; Spencer, R. A.; Khalaf, I. A.; Gilbert, L.; El-Bassel, N.; Silverman, J. G.; Raj, A. 2017. "The influence of Family Violence and Child Marriage on Unmet Need for Family Planning in Jordan." Journal of Family Planning and Reproductive Health Care 43 (2):105-12. doi: 10.1136/jfprhc-2014-101122.

DeJong, Jocelyn, Rana Jawad, Iman Mortagy, and Bonnie Shepard. 2005. "The Sexual and Reproductive Health of Young People in the Arab Countries and Iran." Reproductive Health Matters 13 (25):49-59. doi: 10.1016/S0968-8080(05)25181-9.

DeJong, Jocelyn, Bonnie Shepard, Farzaneh Roudi-Fahimi, and Lori Ashford. 2007. "Young people's sexual and reproductive health in the Middle East and North Africa." Reprod Health 14 (78):8.

Department of Statistics [Jordan]. 2016. "2015 National Census Report." Accessed 10 March 2019. http://dosweb.dos.gov.jo/category/unemployment-rate/.

Department of Statistics [Jordan] and ICF International. 2013. "Jordan Population and Family Health Survey 2012." In. Calverton, MD, USA: Department of Statistics and ICF International.

Dialmy, Abdessamad. 2005. "Sexuality in Contemporary Arab Society." Social Analysis 49 (2):16-33.

Ercevik Amado, Liz. 2009. "Sexuality and Sexual Rights in Muslim Societies." Development 52 (1):59-63. doi: 10.1057/dev.2008.85.

Gausman, Jewel, Danielle Lloyd, Thomas Kallon, SV Subramanian, Ana Langer, and S Bryn Austin. 2019. "Clustered Risk: An Ecological Understanding of Sexual Activity among Adolescent Boys and Girls in Two Urban Slums in Monrovia, Liberia." Social Science and Medicine. 
Gausman, Jewel, Areej Othman, Abla Amawi, and Ana Langer. 2019. "Child marriage in the Arab world." The Lancet 394 (10201):825-6.

Gausman, Jewel, Areej Othman, Iqbal Lutfi Hamad, Maysoon Dabobe, Insaf Daas, and Ana Langer. 2019. "How do Jordanian and Syrian youth living in Jordan envision their sexual and reproductive health needs? A concept mapping study protocol." BMJ open 9 (1):e027266.

Hart, Jason. 2008. "Dislocated Masculinity: Adolescence and the Palestinian Nation-In-Exile." Journal of Refugee Studies 21 (1):64-81.

Higher Population Council. 2017. "دراسة زواج القاصرات في الاردن:" In. Amman, Jordan: Higher Population Council.

Hikmat, Ruba. 2017. "A Study On Child Marriage in Jordan." Higher Population Council-Jordan. Accessed December 4:2017.

Inhorn, Marcia C. 2012. The New Arab man: Emergent Masculinities, Technologies, and Islam in the Middle East: Princeton University Press.

Inhorn, Marcia C, and Emily A Wentzell. 2011. "Embodying Emergent Masculinities: Men Engaging with Reproductive and Sexual Health Technologies in the Middle East and Mexico." American Ethnologist 38 (4):801-15.

Iris, Madelyn, John W Ridings, and Kendon J Conrad. 2009. "The Development of a Conceptual Model for Understanding Elder Self-Neglect." The Gerontologist 50 (3):303-15.

Jackson, Kristin M, and William MK Trochim. 2002. "Concept Mapping as an Alternative Approach for the Analysis of Open-Ended Survey Responses." Organizational Research Methods 5 (4):307-36.

James, Leah, Annie Sovcik, Ferdinand Garoff, and Reem Abbasi. 2014. "The Mental Health of Syrian Refugee Children and Adolescents." Forced Migration Review 47.

Kane, Mary, and William MK Trochim. 2007. Concept Mapping for Planning and Evaluation. Vol. 50: Sage.

Khalaf, Inaam, Fathieh Abu Moghli, and Erika Sivarajan Froelicher. 2010. "Youth-Friendly Reproductive Health Services in Jordan from the Perspective of the Youth: A Descriptive Qualitative Study." Scandinavian Journal of Caring Sciences 24 (2):321-31.

Mrayan, L, and F Cornish. 2015. "“It's our Culture" and the Transition to Parenthood in Jordan: A Descriptive Qualitative Study." J Women's Health Care 4 (254):2167-0420.1000.

Mumtaz, G, N Hilmi, W McFarland, RL Kaplan, and FA Akala. 2011. "Are HIV Epidemics among Men Who Have Sex with Men Emerging in the Middle East."

Obermeyer, Carla Makhlouf. 2000. "Sexuality in Morocco: Changing Context and Contested Domain." Culture, health \& sexuality 2 (3):239-54.

Obermeyer, Carla Makhlouf, Sarah Bott, and Anniebelle J. Sassine. 2015. "Arab Adolescents: Health, Gender, and Social Context." Journal of Adolescent Health 57 (3):252-62. doi: https://doi.org/10.1016/j.jadohealth.2015.01.002.

Okour, Abdelhakeem M, and Raja Badarneh. 2011. "Spousal Violence Against Pregnant Women from a Bedouin Community in Jordan." Journal of Women's Health 20 (12):1853-9.

Oksanen, Jari, F. Guillaume Blanchet, Roeland Kindt, Pierre Legendre, Peter R. Minchin, R. B. O'Hara, Gavin L. Simpson, et al. 2016 "Vegan: Community Ecology Package." R package version 2.3-4. 
Schoemaker, J, A Yassa, S Farah, LK Qardan, LS Jaroudi, and MN El-Sarayrah. 2001. "Jordanian Youth Survey: Knowledge Attitudes and Practices on Reproductive Health and Life Planning. Final Report."

Sexual and Gender-Based Violence Sub-Working Group. 2015. "Sexual and Gender-Based Violence in Refugees in Jordan." In. Amman, Jordan: UNFPA, UNHCR.

Uhlmann, Allon J. 2005. "Introduction: Reflections on the Study of Sexuality in the Middle East and North Africa." Social Analysis 49 (2):3-15.

UNICEF. 2015. "Equity for Children \& Adolescents in Jordan: A Country Profile." In.

World Health Organization. 2012. "Mental Health and Pyschosocial Support for Conflict-Related Sexual Violence: Principles and Interventions: Summary." In.: World Health Organization. 
Table 1: Participant Characteristics by Gender

\begin{tabular}{|c|c|c|c|}
\hline & Male & Female & Total \\
\hline Individual Characteristics & $(n=121)$ & $(n=150)$ & $(n=271)$ \\
\hline \multicolumn{4}{|l|}{ Age $(\%(n))$} \\
\hline 15 years & $26.45(32)$ & 39.33 (59) & $33.58(91)$ \\
\hline 16 years & $23.14(28)$ & $20.00(30)$ & $21.4(58)$ \\
\hline 17 years & $18.18(22)$ & $10.67(16)$ & $14.02(38)$ \\
\hline 18 years & $13.22(16)$ & 12.67 (19) & $12.92(35)$ \\
\hline 19 years & $18.18(22)$ & $14.00(21)$ & $15.87(43)$ \\
\hline Missing & $0.83(1)$ & $3.33(5)$ & $2.21(6)$ \\
\hline \multicolumn{4}{|l|}{ Location (\% (n)) } \\
\hline Amman & $18.18(22)$ & $26(39)$ & $22.51(61)$ \\
\hline Irbid & $33.88(41)$ & $25.33(38)$ & $29.15(79)$ \\
\hline Mafraq & $25.62(31)$ & $26(39)$ & $25.83(70)$ \\
\hline Zarqa & $22.31(27)$ & $22.67(34)$ & $22.51(61)$ \\
\hline \multicolumn{4}{|l|}{ Nationality (\% (n)) } \\
\hline Jordanian & $53.72(65)$ & $54.67(82)$ & $54.24(147)$ \\
\hline Syrian & $46.28(56)$ & $45.33(68)$ & $45.76(124)$ \\
\hline \multicolumn{4}{|l|}{ Marital Status (\% (n)) } \\
\hline Married & $0.83(1)$ & $0.67(1)$ & $0.74(2)$ \\
\hline Not married & $93.39(113)$ & $95.33(143)$ & $94.46(256)$ \\
\hline Prefer not to answer & $4.96(6)$ & $2.00(3)$ & $3.32(9)$ \\
\hline Missing & $0.83(1)$ & $2.00(3)$ & $1.48(4)$ \\
\hline
\end{tabular}

Currently Attending School (\%

(n))

$\begin{array}{llll}\text { Yes } & 36.36(44) & 49.33(74) & 43.54(118) \\ \text { No } & 60.33(73) & 40.67(61) & 49.45(134) \\ \text { Missing } & 3.31(4) & 10(15) & 7.01(19)\end{array}$


Table 2: Young Men's and Women's Importance Scores and Bridging Values by Nationality

\begin{tabular}{|c|c|c|c|c|c|c|c|}
\hline \multirow[t]{3}{*}{ Cluster names and items } & \multicolumn{6}{|c|}{ Importance } & \multirow{3}{*}{$\begin{array}{l}\text { Bridging } \\
\text { Value } \\
\text { (Mean) } \\
\end{array}$} \\
\hline & \multicolumn{2}{|l|}{ Combined } & \multicolumn{2}{|l|}{ Syrian } & \multicolumn{2}{|l|}{ Jordanian } & \\
\hline & Mean (SD) & Rank $^{\mathrm{a}}$ & $\begin{array}{l}\text { Mean } \\
\text { (SD) }\end{array}$ & $\begin{array}{l}\text { Rank } \\
\text { a }\end{array}$ & Mean (SD) & $\begin{array}{l}\text { Rank } \\
\text { a }\end{array}$ & \\
\hline \multicolumn{8}{|l|}{ Young women } \\
\hline 1: Wishes & 2.86 & 8 & 2.88 & 4 & 3.26 & 4 & 0.32 \\
\hline 4: Conservative society & 3.00 & 3 & 2.85 & 4 & 3.04 & 5 & 0.60 \\
\hline $\begin{array}{l}\text { 5: Obtaining information from parents and other credible } \\
\text { sources }\end{array}$ & 2.92 & 7 & 2.97 & 2 & 3.28 & 3 & 0.25 \\
\hline 6: Violence and unhealthy marriage & 2.77 & 9 & 2.80 & 6 & 3.02 & 6 & 0.50 \\
\hline 7: Fears & 2.99 & 4 & 2.76 & 7 & 2.96 & 7 & 0.24 \\
\hline 1: Educational and informational sources & 2.75 & 2 & 2.71 & 3 & 2.80 & 2 & 0.92 \\
\hline 2: Socially unacceptable behaviours and shame & 2.31 & 6 & 2.36 & 6 & 2.26 & 6 & 0.09 \\
\hline 3: Fears & 2.75 & 3 & 2.73 & 1 & 2.69 & 3 & 0.20 \\
\hline 4: SRH information, needs and concerns & 2.59 & 5 & 2.53 & 5 & 2.65 & 4 & 0.24 \\
\hline 5: Factors influencing SRH & 2.26 & 7 & 2.36 & 7 & 2.17 & 7 & 0.67 \\
\hline 6: Transition to adulthood and puberty & 2.61 & 4 & 2.56 & 4 & 2.65 & 5 & 0.58 \\
\hline 7: Needs for a safe and healthy marital life & 2.84 & 1 & 2.72 & 2 & 2.95 & 1 & 0.73 \\
\hline
\end{tabular}

a Rank indicates a cluster's relative ranking in terms of average importance with regard to the clusters. A ranking of 1 indicates that a cluster had the highest average importance score of all the items. 
Figure 1: Young Women's Concept Map
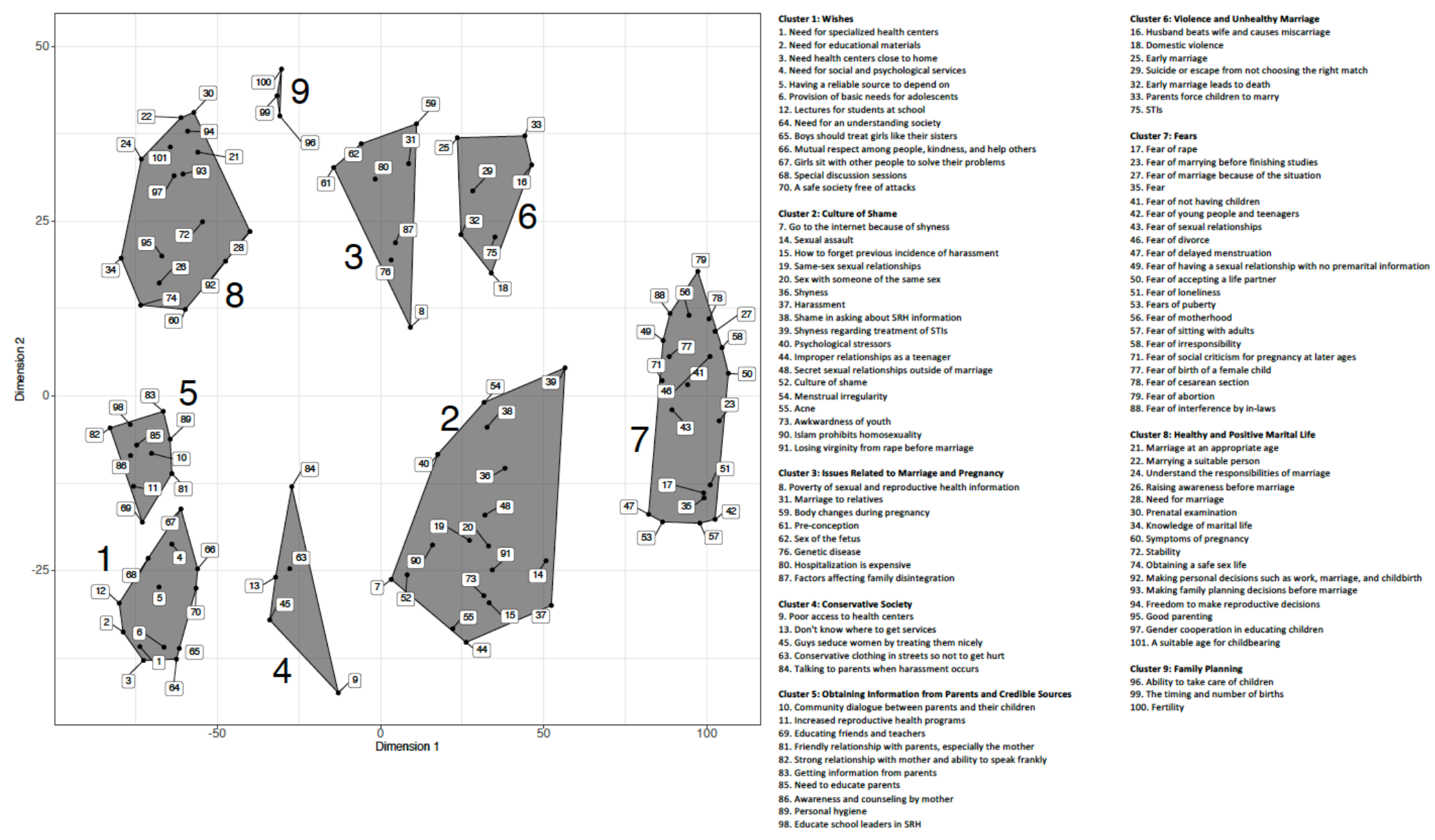
Figure 2: Young Men’s Concept Map
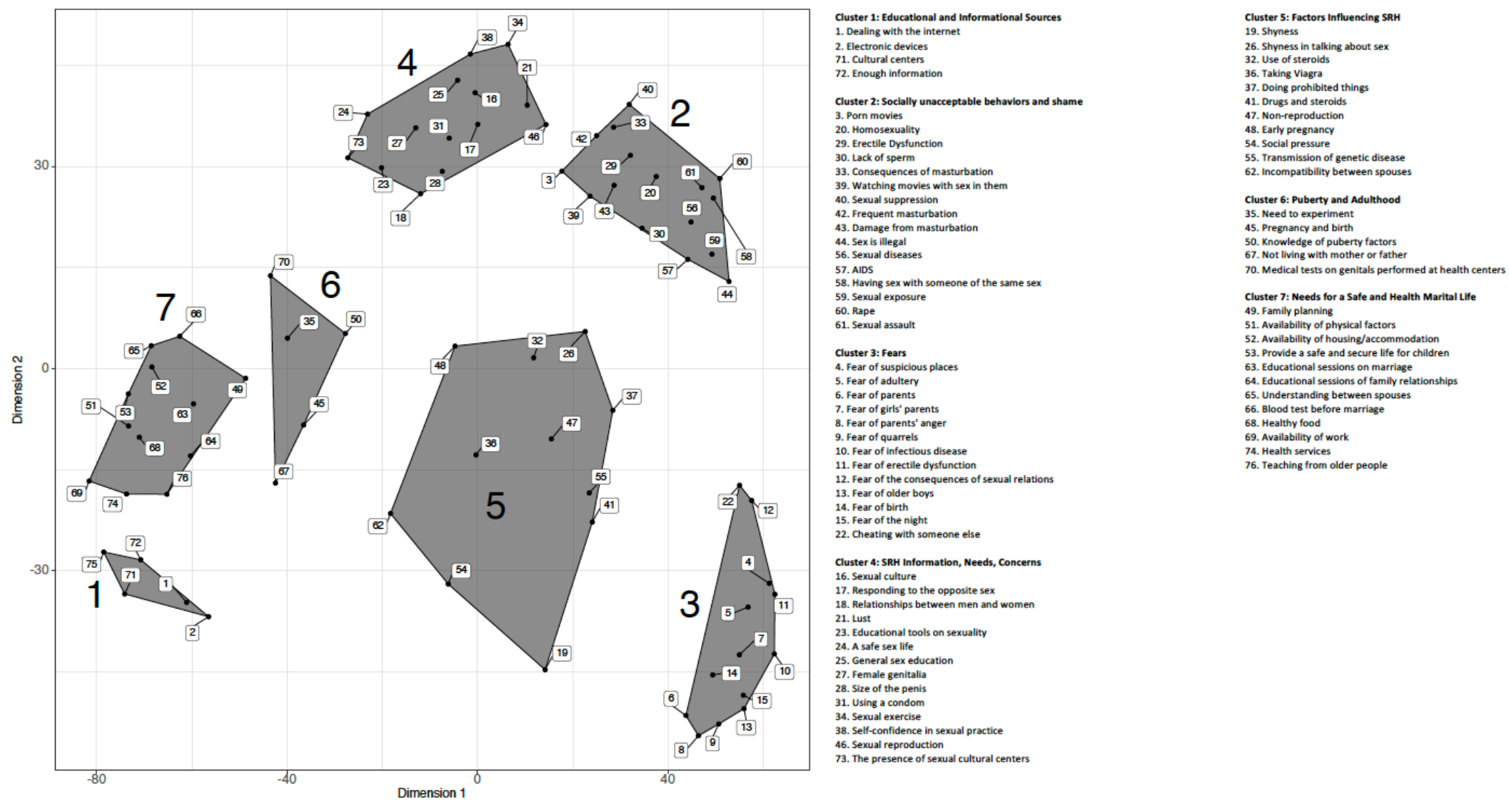